\title{
La mortalidad por suicidios: México 1990-2001
}

\author{
Esteban Puentes-Rosas, ${ }^{1}$ Leopoldo López-Nieto ${ }^{1}$ \\ y Tania Martínez-Monroy ${ }^{1}$
}

Forma de citar

Puentes-Rosas E, López-Nieto L, Martínez-Monroy T. La mortalidad por suicidios: México 1990-2001. Rev Panam Salud Publica. 2004;16(2):102-9.

RESUMEN Objetivo. Describir la mortalidad por suicidios en México en 2001, así como los principales cambios en los métodos de suicidio y en la estructura por edades y sexos que se han presentado desde 1990, tanto a escala nacional como por entidad federativa.

Métodos. Para este estudio descriptivo se utilizaron como fuentes de información los registros oficiales de mortalidad del Instituto Nacional de Estadística, Geografía e Informática para el período de 1990 a 2001. Para calcular las tasas de mortalidad se usaron las poblaciones estimadas por el Consejo Nacional de Población en 2002. Se describieron la mortalidad por sexo, por grupo de edad y por entidad federativa, así como los cambios observados en el período de 19902001 en las tasas y los métodos de suicidio. Para estandarizar las tasas se empleó el método directo, y como estándar se tomaron los datos de población del año 2000. Las comparaciones estadísticas de las tendencias según el grupo de edad y el sexo se llevaron a cabo mediante una prueba de paralelismo utilizando el estadígrafo $F$. El nivel de significación estadística de las diferencias en los métodos de suicidio se determinó mediante la prueba de ji al cuadrado.

Resultados. Durante el año 2001 se registraron 3784 suicidios (3 110 de ellos en hombres y 674 en mujeres), lo que representa una tasa de 3,72 muertes por suicidios por cada 100000 habitantes, en una población total nacional de 101,8 millones de habitantes. Los estados con mayor mortalidad por suicidios fueron Campeche y Tabasco (9,68 y 8,47 por 100000 habitantes, respectivamente). Las menores tasas se observaron en Chiapas y el Estado de México (1,03 y 1,99 por 100000 habitantes, respectivamente). En 2001, la mortalidad por suicidios por 100000 habitantes fue de 6,14 en hombres y de 1,32 en mujeres. El mayor incremento por grupo de edad se observó en las mujeres de 11-19 años (de 0,8 en 1990 a 2,27 por 100000 personas en 2001). En los hombres, el aumento más pronunciado se observó en el grupo de 11-19 años (de 2,6 en 1990 a 4,5 por 100000 personas en 2001). La tasa más alta (13,62 por 100000 personas) se observó en los hombres de más de 65 años. Se observaron cambios en los métodos de suicidio, y el ahorcamiento es el medio más frecuentemente utilizado actualmente tanto por hombres como por mujeres.

Conclusiones. El suicidio es un problema creciente cuyo control exige acciones inmediatas. El incremento acelerado de este fenómeno, particularmente en hombres y mujeres jóvenes, debe ser una llamada de atención para implementar a la brevedad medidas tendientes a disminuir su magnitud.

Palabras clave Suicidio, mortalidad, tasa de mortalidad, México.

1 Dirección General de Evaluación del Desempeño, Secretaría de Salud, México, D.F., México. La correspondencia debe dirigirse a Esteban Puentes Rosas, Reforma 450, piso 12, Colonia Juárez, 06600, México, D.F., México. Correo electrónico: epuentes @salud.gob.mx
El suicidio es un acto complejo en el que se pueden identificar al menos tres elementos: ocurre un deceso, la muerte se ve ocasionada por la misma persona que muere, y el acto es intencional, es decir, la persona suicida está plenamente consciente de las consecuencias de su acto (1). La mortalidad por suicidios se ha convertido en un problema de prioridad para la salud pública, 
tanto a escala nacional como internacional. A pesar de que la muerte por suicidio es solo la parte más visible de un problema que tiene amplias conexiones sociales, psicológicas y personales, y de que frecuentemente se ha argumentado que los registros habituales de suicidios tienden a subestimar el problema (2), la mayor parte de las conclusiones que se pueden sacar a partir de las cifras oficiales son esencialmente correctas (3).

El incremento en la frecuencia de suicidios está asociado con diversos factores que varían en dependencia del grupo de edad estudiado. Entre los jóvenes influyen la depresión, el aumento del consumo de drogas y de alcohol; en los adultos mayores, las enfermedades que generan gran dolor o discapacidad; y en los grupos de edad laboralmente activos, otros factores de índole laboral y económica $(4,5)$. Un estudio realizado en adolescentes que residían en la ciudad de México mostró una asociación entre la ideación suicida en los adolescentes y vivir en hogares donde estaba presente solo uno de los padres, situación que es cada vez más frecuente en México (6).

Durante mucho tiempo, México presentó una de las tasas de mortalidad por suicidios más bajas de América Latina. Sin embargo, en los últimos años la mortalidad por esta causa se ha incrementado progresivamente en este país a un ritmo más acelerado que en otros países de la Región (7).

Teniendo en cuenta que el trabajo publicado más recientemente sobre la mortalidad por suicidios en México se basa en datos obtenidos hasta 1994 (8), se hace necesario actualizar la información acerca de este tema. El objetivo del presente trabajo fue describir la mortalidad por suicidios en México en 2001, así como los principales cambios en los métodos de suicidio y en la estructura por edades y sexos que se han presentado desde 1990, tanto a escala nacional como por entidad federativa.

\section{MATERIALES Y MÉTODOS}

El presente estudio es de carácter descriptivo y se basa principalmente en la información sobre mortalidad que publica anualmente el Instituto Nacional de Estadísticas, Geografía e Informática (INEGI), institución responsable de las estadísticas oficiales en México. Adicionalmente se consultaron los informes sobre los intentos de suicidio en los anuarios que publica periódicamente el INEGI (9) y en la base de datos del Sistema Epidemiológico y Estadístico de Defunciones (SEED), bajo la responsabilidad de la Secretaría de Salud.

Con la información obtenida de estas fuentes se procedió a analizar las tasas de mortalidad por suicidios en los años 1990 y 2001. La descripción se realizó según las 32 entidades federativas, por sexo y por los grupos de edad de 0 a 10 , de 11 a 19 , de 20 a 39, de 40 a 64 y de 65 años o más. De esta forma se podían distinguir las muertes en adolescentes y en los adultos mayores, que son los grupos donde se han observado el mayor incremento en la frecuencia de suicidios y la mayor tasa, respectivamente. No se analiza la mortalidad en menores de 11 años, ya que las cifras observadas en este grupo son muy bajas y no permiten hacer estimaciones lo suficientemente robustas. Todas las tasas se expresaron en muertes por 100000 personas del sexo y grupo de edad en cuestión, a partir de las estimaciones de población obtenidas por el Consejo General de Población.

Se analizaron los principales métodos de suicidio en el país, según el sexo y el grupo de edad, así como los cambios observados en los métodos de suicidio en cada grupo estudiado.

La comparación estadística de las tendencias según el grupo de edad y el sexo se llevó a cabo mediante una prueba de paralelismo utilizando el estadígrafo $F$ (10). El nivel de significación estadística de las diferencias en los métodos de suicidio se evaluó mediante la prueba de ji al cuadrado. Para evaluar el efecto que el cambio demográfico ha tenido en el incremento de la mortalidad por suicidios se estandarizaron los datos por el método directo, utilizando la estructura poblacional de México en el año 2000 como población de referencia. Se escogió un nivel de significación estadística de 0,05 .

\section{RESULTADOS}

\section{Mortalidad por suicidios según el sexo}

Durante el año 2001 se registraron 3784 suicidios (3 110 de ellos en hombres y 674 en mujeres), lo que representa una tasa de 3,72 muertes por suicidio por cada 100000 habitantes, en una población total nacional de 101,8 millones de habitantes. Los estados con mayor mortalidad por suicidios ese año fueron Campeche y Tabasco, con tasas de 9,68 y 8,47 por 100000 habitantes, respectivamente. Las menores tasas se observaron en Chiapas y el Estado de México, con valores de 1,03 y 1,99 por 100000 habitantes, respectivamente.

La mortalidad por suicidios en el país fue notablemente más baja en mujeres (1,32 por 100000 habitantes) que en hombres (6,14 por 100000 habitantes). Dada esta notable diferencia, se compararon las tasas observadas tanto en el nivel nacional como en el estadual. Los estados con mayor mortalidad por suicidios en mujeres fueron Campeche, Yucatán y Tabasco, todos ubicados en el sureste del país, con tasas de 4,48, 2,80 y 2,04 por 100000 habitantes, respectivamente. La tasa de mortalidad por suicidios en mujeres de Campeche durante 2001 fue más de tres veces mayor que la tasa nacional en mujeres, mientras que los estados con menor mortalidad por esta causa en mujeres fueron Chiapas, Sinaloa y Baja California Sur, con tasas de $0,53,0,53$ y 0,91 por 100000 habitantes, respectivamente (figura 1).

En el caso de los hombres, los estados con mayor mortalidad por suicidios fueron Tabasco, con 14,81, Campeche, con 14,73, y Sonora, con 12,69, todas por 100000 habitantes; mientras que las entidades federales con menor mortalidad por esta causa en hombres fueron Chiapas, el Estado de México y Guerrero, cuyas tasas fueron de 1,53, 2,98 y 3,70 por 100000 habitantes, respectivamente (figura 1). Como puede observarse, en Tabasco la tasa de mortalidad por suicidios en hombres es más del doble de la tasa nacional para ese sexo, mientras que en Chiapas, 
FIGURA 1. Mortalidad por suicidios en hombres y mujeres (por 100000 habitantes del sexo correspondiente), por entidad federativa. México, 2001

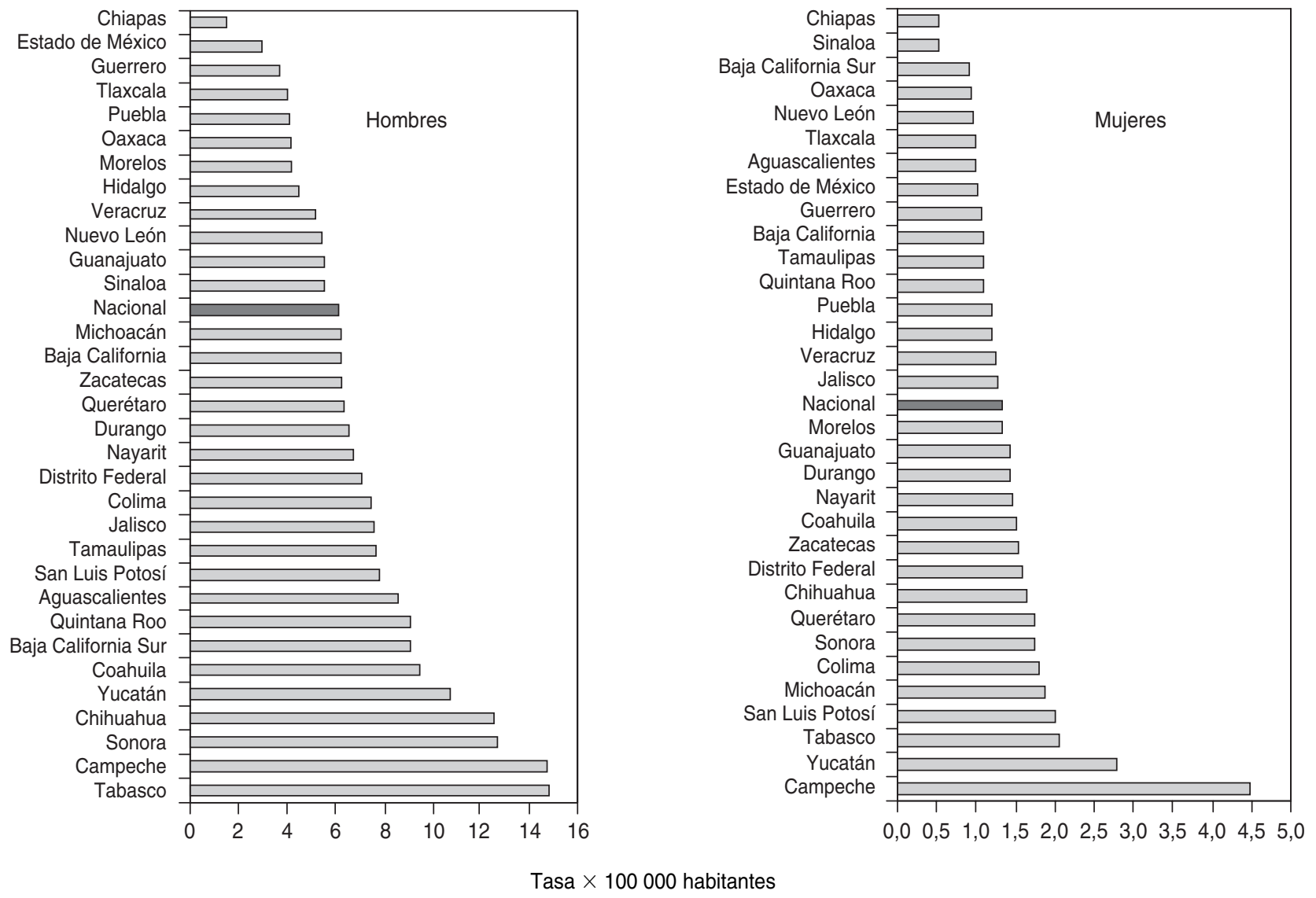

estado con muy bajo nivel de desarrollo y colindante con Tabasco, la tasa de suicidios en hombres es apenas la cuarta parte de la tasa nacional.

Las muertes por suicidio no muestran una distribución uniforme en el tiempo. En general parecen concentrarse en los meses de abril y mayo, aunque en las mujeres existe un pico en diciembre, mes en el que se producen $10,2 \%$ de las defunciones por esta causa. La distribución temporal observada difiere significativamente $(P<$ $0,01)$ de la esperada con una distribución uniforme a lo largo del año (datos no mostrados).

El lugar donde se cometen los suicidios con más frecuencia es el hogar, independientemente del sexo, con una frecuencia de $80 \%$ en los hombres y de $89 \%$ en las mujeres, mientras que la vía pública es el lugar donde ocurren menos suicidios ( $8 \%$ en hombres y $4 \%$ en mujeres).

\section{Mortalidad por suicidios según el grupo de edad}

En el año 2001, la mayor mortalidad por suicidios en hombres se observó en el grupo de 65 años o más, con una tasa de 13,62 por 100000 . A escala estadual, la cifra más alta se observó en Tabasco, al sureste del país, también en el grupo de mayor edad, con una tasa de 54 muertes por 100 000. Ese estado también tiene la mayor tasa de suicidios en el grupo de 40 a 64 años y se encuentra entre las cinco entidades federales con las mayores tasas en los otros grupos de edad estudiados.

En las mujeres, la tasa de suicidios más alta se observó en el grupo de 11 a 19 años de edad, con un valor de 2,27 por 100000 . En este grupo de edad, los suicidios representaron $6,1 \%$ del total de defunciones y constituyeron la segunda causa de muerte, solamente inferior a la mortalidad por accidentes de tránsito. Entre los estados, la mayor mortalidad se observó en Campeche, también en el sureste mexicano, particularmente en el grupo de 40 a 64 años de edad, con una tasa de 7,8 por 100 000. Este estado se ubicó entre las tres entidades federales con las mayores tasas de mortalidad por suicidios en cualquiera de los grupos estudiados.

Las tasas más bajas por grupo de edad según el sexo fueron la de los hombres de 11 a 19 años (4,53 por $100000)$ y la de las mujeres de 65 años o más (1,16 por 100 000). Las muertes por suicidio en hombres entre los $11 \mathrm{y}$ 
19 años de edad representaron 6,4\% de todas las defunciones en hombres de ese grupo de edad.

En el país se notificaron nueve muertes por suicidio en niños menores de 11 años. Siete de estas muertes ocurrieron en varones. Jalisco, estado del occidente del país que se caracteriza por una fuerte migración de sus zonas rurales, fue el único estado donde hubo más de un caso de suicidio en menores de 10 años (dos defunciones).

\section{Cambios en la mortalidad por suicidios en el período de 1990-2001}

En términos absolutos, los suicidios aumentaron de 1930 en 1990 a 3784 en el año 2001. El número de suicidios aumentó de 281 a 674 en mujeres y de 1646 a 3110 en hombres (3 notificaciones de suicidio de 1990 no informaban el sexo del suicida). Estos incrementos representaron un cambio en la tasa de mortalidad por suicidios en hombres de 3,90 muertes por 100000 individuos en 1990 a 6,14 en 2001 (figura 2).

La estandarización de las tasas mostró que el efecto del envejecimiento de la población sobre este incremento ha sido mínimo. El aumento de las tasas estandarizadas fue de aproximadamente 0,16 muertes por 100000 habitantes por año, frente a un incremento de 0,20 anual en las tasas observadas. En el caso de las mujeres, la tasa observada en 2001 fue el doble de la observada en 1990. Aunque el número de suicidios en mujeres es bastante más bajo que en los hombres, su incremento relativo ha sido mayor $\mathrm{y}$, de acuerdo con el análisis de las tasas estandarizadas, ha sufrido menos el efecto de los cambios demográficos en el período estudiado. Cabe destacar que entre 1993 y 1997 hubo un aumento acelerado de 0,1 muertes por 100000 habitantes anuales en la tasa de mortalidad por suicidios en mujeres, frente a un aumento promedio de 0,05 por año entre 1990 y 2001 (figura 2).

El mayor incremento en las tasas de suicidios, calculadas por sexo y grupo de edad, se observó en las mujeres de 11 a 19 años, en quienes la tasa observada ascendió de 0,8 en 1990 a 2,27 por

FIGURA 2. Tasas observadas y estandarizadas ${ }^{a}$ de mortalidad por suicidios en hombres y mujeres (por 100000 habitantes del sexo correspondiente), por año. México, 1990-2001
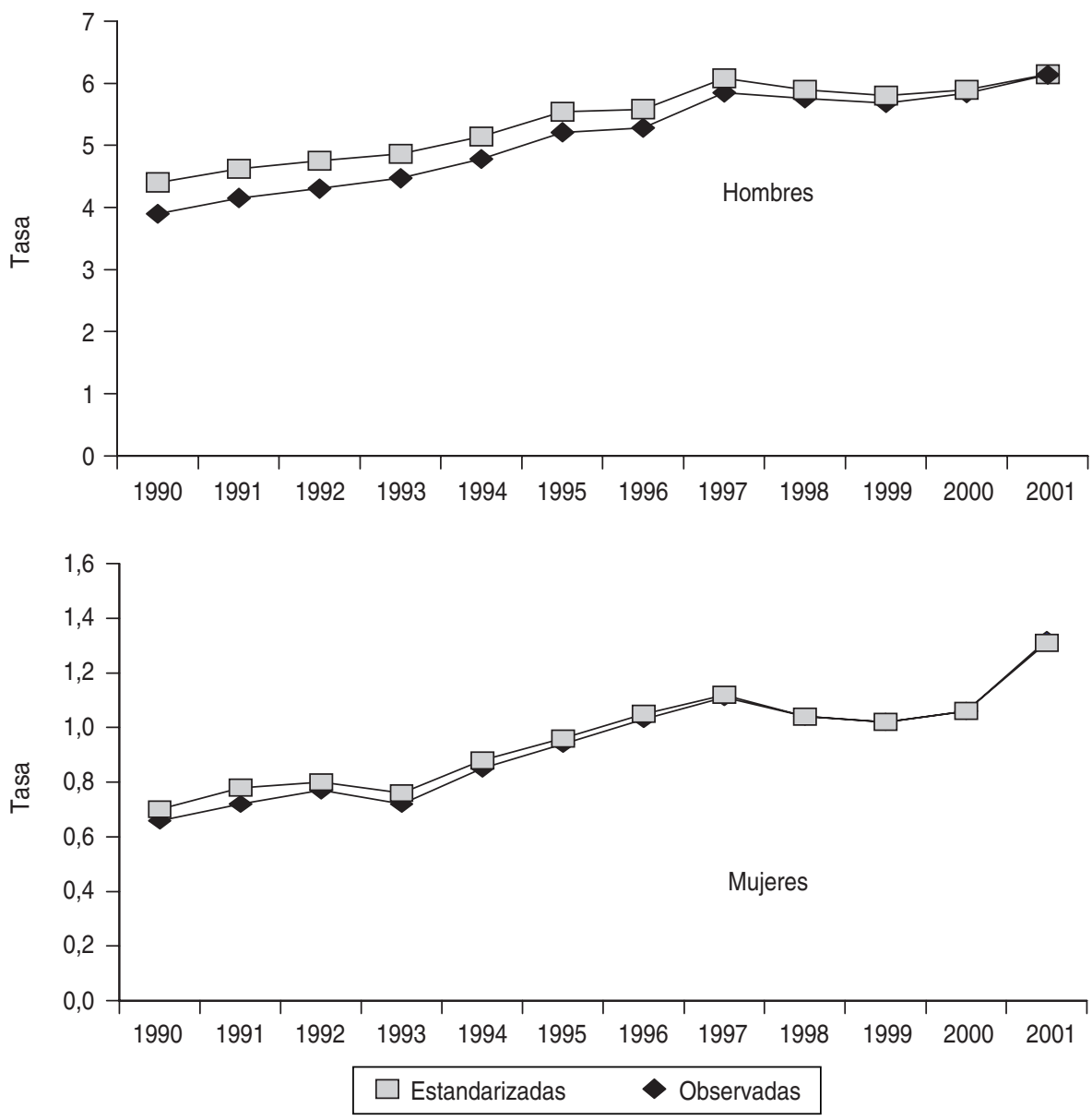

a Estandarizadas por el método directo utilizando como estándar la población de México en el año 2000.

100000 habitantes en 2001 (figura 3). En los hombres, el mayor incremento se encontró también entre los adolescentes y los adultos jóvenes. Entre 1990 y 2001, la tasa aumentó de 2,6 a 4,5 por 100000 en el grupo de 11 a 19 años y de 6,4 a 9,5 en el de 20 a 39 años (figura 4).

Por su parte, la mortalidad en las mujeres de 40 a 64 años y de 65 años o más fue ligeramente mayor en 2001 que en 1990, aunque no se observó una tendencia estable durante el período estudiado. La comparación de las pendientes (coeficientes $\beta$ de las rectas de regresión) demostró que existen diferencias estadísticamente significativas entre los incrementos de las tasas de mortalidad de los diferentes grupos estudiados y entre las pendientes co- rrespondientes a los hombres y las mujeres $(P<0,05)$.

Los incrementos más marcados en la mortalidad por suicidios en hombres se observaron en los estados de Chihuahua, Sonora y Aguascalientes, donde las tasas se elevaron en el período estudiado de 5,18 a 12,53 , de 6,22 a 14,81 y de 3,27 a 8,59 por 100 000, respectivamente. En Colima, Tabasco y Chiapas, las tasas de mortalidad por suicidios entre 1990 y 2001 disminuyeron en 1,94, 1,44 y 0,95 muertes por 100000 habitantes, respectivamente.

En el caso de las mujeres, las variaciones más importantes se encontraron en Campeche y Yucatán, donde las tasas de suicidios aumentaron en 3,03 y 1,54 muertes por 100000 habitantes, 
FIGURA 3. Mortalidad por suicidios en mujeres (por 100000 habitantes de sexo femenino), según grupo de edad y año. México, 1990-2001

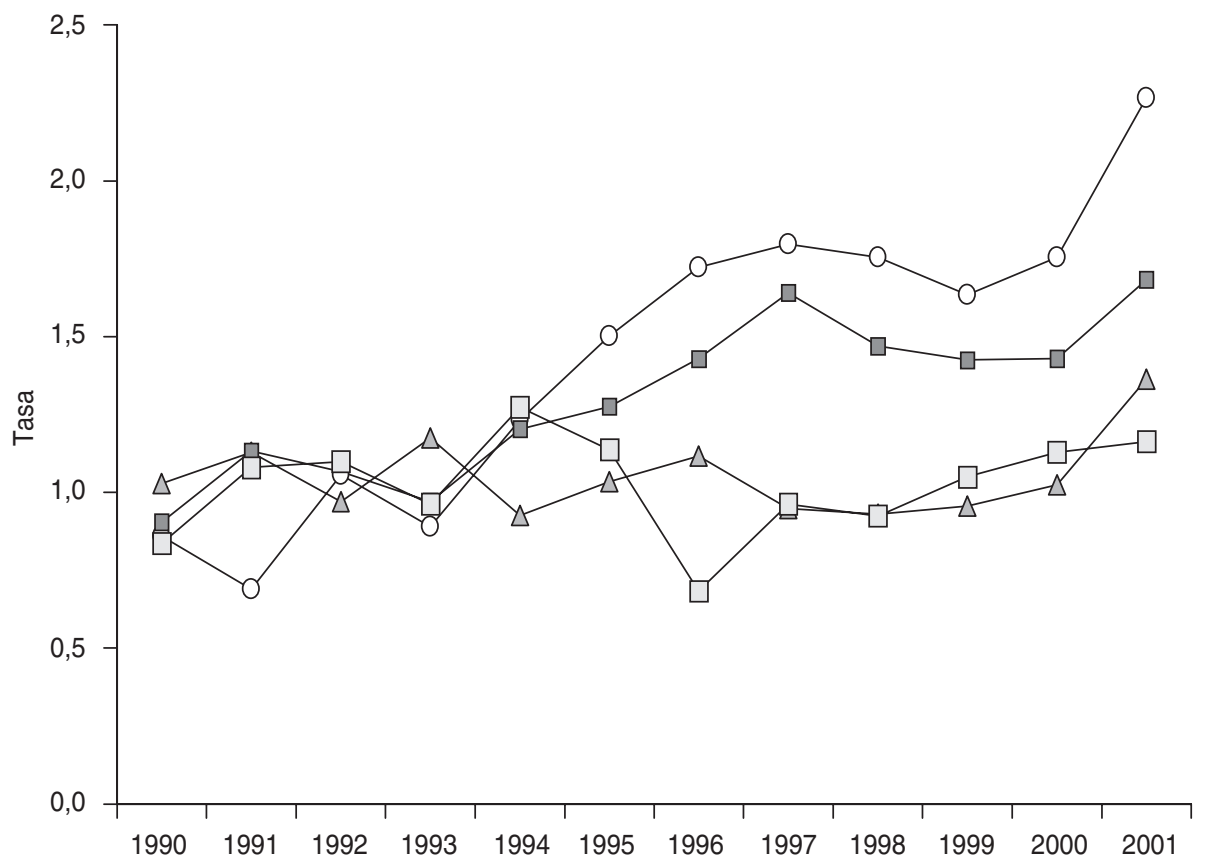

FIGURA 4. Mortalidad por suicidios en hombres (por 100000 habitantes de sexo masculino), según grupo de edad y año. México, 1990-2001

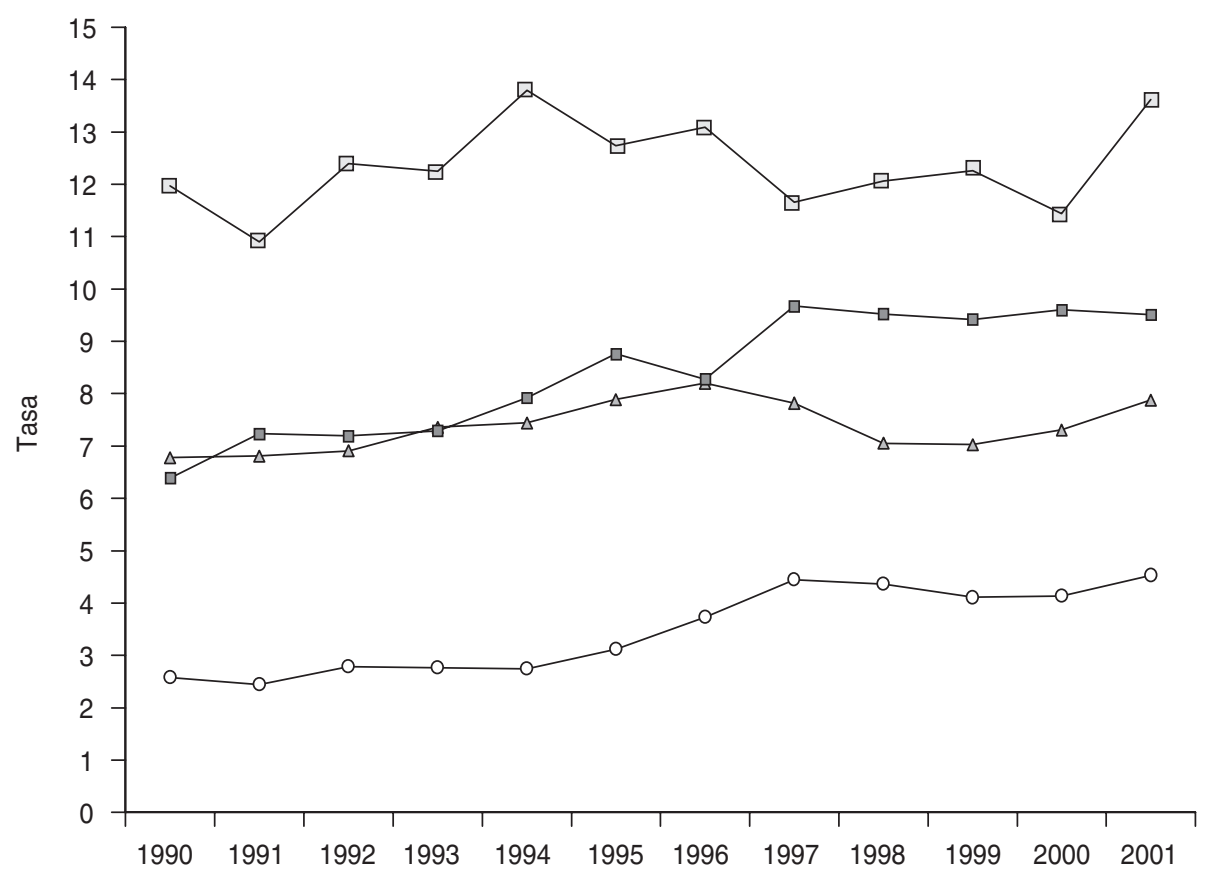

Edad (años): $\multimap-11-19 \quad \rightarrow-20-39 \quad \rightarrow-40-64 \quad \neg \square-65+$ respectivamente. Solo se observaron disminuciones en las tasas de Quintana Roo y Tabasco (disminuyeron en 0,67 y 0,11 muertes por 100000 habitantes, respectivamente).

\section{Métodos de suicidio}

Los métodos de suicidio variaron en dependencia del sexo y de la edad del suicida, y se observaron cambios entre 1990 y 2001. En 1990, el mayor número de suicidios fue por ahorcamiento $(49,4 \%)$, mientras que $28,5 \%$ de las defunciones se produjeron por disparo con arma de fuego y $13,4 \%$ por ingestión de sustancias tóxicas. Ese año se observó que el método más frecuentemente utilizado variaba según el sexo: $51,9 \%$ de los hombres que cometieron suicidio lo hicieron mediante ahorcamiento, mientras que $39,5 \%$ de las mujeres suicidas recurrieron al envenenamiento, ya sea por consumo de productos agroquímicos o plaguicidas, o por sobredosis de fármacos de muy variable naturaleza, como analgésicos, neurofármacos y narcóticos, entre otros, aunque estos últimos fueron los más frecuentes (cuadro 1).

En 2001, el porcentaje de suicidios por ahorcamiento ascendió a $67,2 \%$, mientras que la utilización de armas de fuego disminuyó a 18,9\% y el envenenamiento a $9,7 \%$. El incremento del porcentaje de muertes por ahorcamiento fue mayor en las mujeres, en quienes la proporción de muertes por esta causa se elevó de $34,2 \%$ en 1990 a $51,3 \%$ en 2001; el segundo método más frecuentemente utilizado por las mujeres según los registros de 2001 fue el envenenamiento (27,3\%). En hombres, los suicidios por ahorcamiento constituyeron el $70,2 \%$ de todos los suicidios, mientras que el disparo con arma de fuego fue el segundo método más utilizado (19,8\%) (cuadro 1).

Por otro lado, los mecanismos de suicidio también han variado en el período de estudio, aunque esas diferencias no están respaldadas estadísticamente $(0,05<P<0,10)$. Las diferencias en los métodos de suicidio entre hombres y mujeres en 2001 fueron estadísticamente significativas $(P<0,01)$. 
CUADRO 1. Métodos de suicidio utilizados (en porcentajes del total por año) por sexo. México, 1990 y 2001

\begin{tabular}{lrrrrr}
\hline \multirow{2}{*}{\multicolumn{1}{c}{ Método de suicidio }} & \multicolumn{2}{c}{ Hombres $^{\mathrm{a}}$} & & \multicolumn{2}{c}{ Mujeres $^{\mathrm{a}}$} \\
\cline { 2 - 3 } \cline { 6 - 7 } & 1990 & 2001 & & 1990 & 2001 \\
\hline Ahorcamiento & 51,9 & 70,2 & & 34,2 & 51,3 \\
Disparo de arma de fuego & 30,8 & 19,8 & & 15,7 & 14,3 \\
Intoxicación/envenenamiento & 9,3 & 5,9 & & 39,5 & 27,5 \\
Otros & 8,0 & 4,1 & & 10,6 & 6,9 \\
\hline
\end{tabular}

${ }^{a}$ Diferencia estadísticamente significativa entre hombres y mujeres $(P<0,01)$.

\section{DISCUSIÓN}

El suicidio es el punto culminante -y el más visible- de una serie de problemas que pueden presentarse juntos o por separado y que pueden deberse a factores económicos, predisposición genética, conflictos familiares, desintegración social o falta de identificación y atención oportuna a los problemas de salud mental, particularmente a la depresión (11). Por lo tanto, es necesario conocer la forma en que las muertes por suicidio evolucionan en el país para poder interpretar cómo diferentes variables afectan a la salud de la población.

La mortalidad por suicidios en México ha aumentado en los últimos años. Aunque en varios grupos de edad la tasa de suicidios se ha mantenido relativamente constante, su aumento en algunos grupos, particularmente en las mujeres adolescentes, alcanza niveles preocupantes.

Entre las variables más estrechamente asociadas con el suicidio se encuentran las enfermedades mentales. Hasta $90 \%$ de las personas que comenten suicidio presentan al menos uno de los diagnósticos psiquiátricos basados en el DSM IV (1). Las enfermedades más frecuentes entre los suicidas son la depresión mayor y la esquizofrenia $(1,4)$. Un estudio realizado en Dinamarca mostró que tener antecedentes de enfermedad mental, particularmente de esquizofrenia, era el factor más fuertemente asociado con la probabilidad de suicidarse (12).

Uno de los aspectos más preocupantes de las muertes por suicidio es su incremento entre los adolescentes y jóve- nes adultos. Como muestran las cifras presentadas, entre 1990 y 2000 la tasa de mortalidad por suicidios se duplicó en el grupo de 11 a 19 años, con un incremento todavía más marcado entre las mujeres. Según diversos autores, el aumento del consumo de drogas entre los jóvenes es un factor estrechamente relacionado con este incremento (5, 13). No obstante, es importante no perder de vista otros factores de tipo social que afectan directamente a la calidad de la vida de los jóvenes y a sus expectativas para el futuro $(12,14)$. Datos proporcionados por la Organización Panamericana de la Salud (OPS), basados en encuestas realizadas entre jóvenes, muestran que hasta $50 \%$ de ellos manifestaron haberse sentido alguna vez tan deprimidos que se preguntaban si valía la pena seguir viviendo. Aproximadamente $20 \%$-ligeramente más entre las mujeresmanifestaron tener un familiar o un amigo que había intentado suicidarse (15). Cualquier estrategia encaminada a disminuir la mortalidad por suicidios debe necesariamente identificar las causas de la desesperanza en los jóvenes, combatir el creciente consumo de drogas e identificar los grupos de alto riesgo.

Un elemento que no ha sido suficientemente explorado en México es la influencia que pueden ejercer los medios de comunicación masiva sobre el comportamiento de las personas que pudieran intentar suicidarse. La difusión imprudente de la información relacionada con las muertes por suicidio, su descripción precisa -incluso con presentación de imágenes reales- y el uso de términos tales como "suicidio exitoso" crean una situación de "moda" o imitación que podría influir en el aumento de la frecuencia de suicidios (16-18). Habitualmente, la información acerca de los suicidios en los medios simplifica excesivamente el problema y los atribuye a factores únicos, usualmente de carácter económico o sentimental. El acto suicida en ocasiones se presenta como un acto heroico y se suele subestimar - si no omitir- el papel que desempeñan las enfermedades mentales en la tendencia al suicidio (19). Aunque también existen opiniones que niegan el impacto de los medios de comunicación en el suicidio (20) o que solo reconocen su influencia en relación con el método mediante el cual se lleva a cabo, se ha documentado la reducción en el número de suicidios e intentos de suicidio cuando los periodistas se han incorporado a las estrategias de prevención $(21,22)$.

Por supuesto, la disponibilidad de medios para efectuar el suicidio es otra variable que debe evaluarse $y$, en la medida de lo posible, controlarse. Las referencias que asocian la disponibilidad de armas de fuego con los suicidios son abundantes $(23,24)$ y la asociación entre el número de suicidios y la disponibilidad de plaguicidas en el medio rural también refleja el hecho de que la accesibilidad de los medios es un factor que debe controlarse. Las diferencias observadas entre los hombres y las mujeres en relación con los métodos de suicidio pueden estar relacionadas precisamente con la disponibilidad de los medios.

La disparidad entre los sexos en la mortalidad por suicidios es un fenómeno ampliamente reconocido en todo el mundo. Según estos resultados, en México los suicidios son 4,6 veces más frecuentes en hombres que en mujeres. Esta diferencia se observa en todos los estados federales del país, aunque con diferentes magnitudes. En Tabasco, por ejemplo, la tasa de suicidios en hombres es 7,3 veces mayor que en mujeres, mientras que en el Estado de México o Chiapas, la mortalidad por suicidios en hombres es solo 3 veces mayor que en mujeres.

Las diferencias entre sexos reflejan aspectos relacionados con el papel de 
cada género. La mortalidad masculina asociada con causas externas está estrechamente vinculada con las actitudes que tradicionalmente se consideran propias de los hombres, como el papel de proveedor y protector y la búsqueda del "éxito" (15). Otros papeles adjudicados al hombre que pueden estar asociados de manera directa con la frecuencia de suicidios son el consumo de drogas y alcohol y el acceso a armas de fuego. No obstante, estas diferencias no explican por qué los intentos de suicidio son más frecuentes entre las mujeres que entre los hombres.

Una diferencia importante observada entre los casos de suicidio en hombres y en mujeres está relacionada con los métodos que se emplean para cometer o intentar cometer el suicidio. Entre los hombres el uso de armas de fuego es más frecuente que entre las mujeres, mientras que estas recurren a sobredosis de medicamentos o sustancias tóxicas con mayor frecuencia, como han demostrado también otros autores (25). Los métodos utilizados para suicidarse tienen diferentes gra- dos de violencia y letalidad y, por lo tanto, condicionan la posibilidad de recibir atención. En el caso de las mujeres, se recibió atención en 30\% de las muertes, y en el de los hombres, solamente en $14 \%$. Las diferencias en la letalidad de los métodos empleados se reflejan también en los registros de intentos de suicidios, según los cuales, $62 \%$ de los intentos correspondieron a envenenamientos o sobredosis de medicamentos y solo $10 \%$ de los que utilizaron este medio murieron. En cambio, $5 \%$ de los intentos de suicidios fueron por disparo de arma de fuego, mientras que $19 \%$ de los suicidas que emplearon este medio murieron (9). De hecho, el incremento en la utilización de métodos más letales para quitarse la vida, particularmente del ahorcamiento, puede ser un factor que esté influyendo en la disminución de la brecha entre hombres y mujeres.

Las diferencias entre los suicidios y los intentos de suicidios no se limitan a los medios y se manifiestan también en la edad de las personas que se suicidaron o intentaron hacerlo. Mientras que los menores de 29 años cometen $63 \%$ de los intentos de suicidio, a este grupo de edad le corresponden $50 \%$ de las muertes por esta causa. La diferencia se hace más notable en el grupo de 60 años o más, al que corresponden solo $2,8 \%$ de los intentos de suicidio, pero $10 \%$ de las muertes.

Estos resultados ponen de manifiesto que el suicidio es un problema creciente que exige medidas de control inmediatas. Además de la pérdida directa de la vida, el suicidio debe considerarse un hecho trágico que acarrea daños psicológicos profundos y prolongados para los familiares y amigos de la víctima, y en algunos casos puede implicar la pérdida del apoyo económico de la familia. No debe subestimarse el daño a la salud, a veces grave, que ocasionan los intentos fallidos de suicidio. En diversos países se han establecido programas para disminuir la carga que significan los suicidios para la salud. Es tiempo de que en México se establezcan las medidas necesarias a fin de controlar este creciente problema.

\section{REFERENCIAS}

1. Maris R. Suicide. Lancet. 2002;360:319-26.

2. Marusic A, Roskar S, Zorko M. Undetermined deaths: are they suicides? Croat Med J. 2003; 44:550-2.

3. Speechley M, Staravsky KM. The adequacy of suicide statistics for use in epidemiology and public health. Can J Public Health. 1991;82: 38-42.

4. Organización Mundial de la Salud. Informe sobre la Salud en el Mundo. Salud mental: nuevos conocimientos, nuevas esperanzas. Ginebra: Organización Mundial de la Salud; 2001. P. 38.

5. México, Secretaría de Salud. Observatorio mexicano en Tabaco: alcohol y otras drogas. México: Secretaría de Salud; 2002.

6. González-Forteza C, Berenzon-Gorn S, TelloGranados AM, Facio-Flores D, Medina-Mora ME. Ideación suicida y características asociadas en mujeres adolescentes. Salud Publica Mex. 1998;40:430-7.

7. Organización Panamericana de la Salud. La Salud en las Américas. Vol. I. Washington, D.C.: OPS; 1998. (Publicación Científica no. 569)
8. Borges G, Rosovsky H, Gómez C, Gutiérrez R. Epidemiología del suicidio en México de 1970 a 1994. Salud Publica Mex. 1996;38:197-206.

9. Instituto Nacional de Estadística, Geografía e Informática. Estadísticas de Intentos de Suicidios y Suicidios. Cuaderno No. 8. Aguascalientes, México: INEGI; 2002.

10. Kleinbaum D, Kupper L, Muller K. Applied regression analysis and other multivariable methods. $2^{\text {nd }}$ edition. Chapter 4 . Belmont, California: Duxbury Press; 1988. Pp. 265-72.

11. Hawton K. A national target for reducing suicide. Br Med J. 1998;317:156-7.

12. Agerbo E, Nordentoft M, Mortensen PB. Familial, psychiatric, and socioeconomic risk factors for suicide in young people: nested case-control study. Br Med J. 2002;325: 74-9.

13. Marzuk P, Tardiff K, Leon A, Hirsch C, Stajic $M$, Portera $L$, et al. Fatal injuries after cocaine use as a leading cause of death among young adults in New York City. N Engl J Med. 1995; 332:1753-7.

14. Straus MA, Cantor GK. Corporal punishment of adolescents by parents: a risk factor in the epidemiology of depression, suicide, alcohol abuse, child abuse, and wife beating. Adolescence. 1994;29:543-61.

15. Organización Panamericana de la Salud. La Salud en las Américas. Vol. I. Washington, D.C.: OPS; 2002. (Publicación Científica no. 587).

16. Schmidtke A, Häfner H. The Werther effect after television films: new evidence for an old hypothesis. Psychol Med. 1988;18:665-76.

17. Gould MS, Shaffer D. The impact of suicide in television movies. Evidence of imitation. N Engl J Med. 1986;315:690-4.

18. Gunnell D. Reporting suicide: the effect of media coverage on patterns of self harm. Br Med J. 1994;308:1446-7.

19. Hawton K, Williams K. Influences of the media on suicide. Br Med J. 2002;325:1374-5.

20. Philips DP, Paight DJ. The impact of televised movies about suicide. A replicative study. $\mathrm{N}$ Eng J Med. 1987;317:809-11.

21. Etzerdorfer E, Sonneck G. Preventing suicide by influencing mass-media reporting. The Viennese experience 1980-1996. Arch Suicide Res. 1998;4:67-74. 
22. Michel K, Frey C, Wyss K, Valach L. An exercise in improving suicide reporting in print media. Crisis. 2000;21:1-10.

23. Sloan JH, Rivara FP, Reay DT, Ferris JA, Kellermann AL. Firearms regulations and rates of suicide. A comparison of two metropolitan areas. N Eng J Med. 1990;322:369-73.
24. Wintemute GJ, Parham C, Beaumont JJ, Wright M, Drake C. Mortality among recent purchasers of handguns. N Engl J Med. 1999; 341:1583-9.

25. Marín-León L, Barros M. Suicide mortality: gender and socioeconomic differences. Rev Saude Publica. 2003;37:357-63.
Manuscrito recibido el 4 de septiembre de 2003. Aceptado para publicación, tras revisión, el 3 de junio de 2004.

ABSTRACT Objective. To describe mortality from suicides in Mexico in 2001, as well as the main changes in the methods used to commit suicide and in trends by age and gender that have been observed since 1990, both for the country as a whole and for each state.

Mortality from suicides: Mexico, 1990-2001
Methods. For this descriptive study we utilized as information sources the official mortality records of the National Institute of Statistics, Geography, and Informatics (Instituto Nacional de Estadística, Geografía e Informática) for the period of 1990 through 2001. To calculate mortality rates we used the populations estimated in 2002 by the National Population Council (Consejo Nacional de Población). Mortality was described by sex, age group, and state, along with the changes seen over the period of 1990 through 2001 in the rates and methods of suicide. We used the direct method to standardize the rates, using as a reference the population data for the year 2000. To make statistical comparisons of the trends by age group and gender we used a test of parallelism utilizing the $F$ statistic. The level of statistical significance of differences in suicide methods was determined with the chi-square test.

Results. During 2001, 3784 suicides were registered (3 110 of them in men and 674 in women), which represents a rate of 3.72 deaths from suicide per 100000 persons, in a total national population of 101.8 million inhabitants. The states with the highest suicide mortality were Campeche and Tabasco (9.68 and 8.47 per 100 000, respectively). The lowest rates were seen in Chiapas and the state of Mexico (1.03 and 1.99 per 100 000, respectively). In 2001, mortality from suicides per 100000 persons was 6.14 in men and 1.32 in women. The greatest increase by age group was seen in women 11-19 years old (from 0.8 per 100000 in 1990 to 2.27 per 100000 in 2001). The largest increase in men also occurred among those 11-19 years old (from 2.6 per 100000 in 1990 to 4.5 per 100000 in 2001). The highest rate (13.62 per 100000 persons) was seen in men over 65 years of age. There were changes in suicide methods, with hanging now being the method most frequently used by both men and women.

Conclusions. Suicide is a growing problem in Mexico, and controlling it requires immediate steps. The rapid increase in this phenomenon, particularly among young men and young women, should produce a call to action to quickly implement measures aimed at reducing the magnitude of suicide.

\begin{tabular}{l} 
FE DE ERRATUM \\
Ferrero F et al. Prevalencia del consumo de tabaco en médicos \\
residentes de pediatría en Argentina. (Rev Panam Salud Publica/ \\
Pan Am J Public Health 2004:15(6):395-399). \\
\hline La redacción llama la atención de los lectores a un error en la versión publicada \\
del artículo señalado. \\
P. 395, tercera línea de la tercera nota al pie de página: Versión equivocada: \\
"Verónica Rodríguez". Versión correcta: "Viviana Rodríguez".
\end{tabular}

